\title{
Many-Worlds QM Tested by Quantum Chemistry: a New Kind of Reaction Rate
}

\author{
Frank J. Tipler \\ Department of Mathematics, Tulane University, New Orleans, LA 70118
}

(Dated: April 8, 2020)

\begin{abstract}
Quantum chemists have recorded a huge number of data points which standard quantum mechanics cannot interpret. Many-Worlds quantum mechanics can. Many-Worlds quantum mechanics differs from standard quantum mechanics because in Many-Worlds, the wave function is a relative density of universes in the multiverse amplitude rather than a probability amplitude. In ManyWorlds, the Born frequencies are approached rather than given a priori. Thus in Many-Worlds, the rate of approach to the final frequencies can be calculated and compared with observation. This is a new type of reaction rate. I show how to use Many-Worlds to analyze the breakup of $\mathrm{Br}_{2}$. Such analysis will experimentally test Many-Worlds fermionic and time-dependent quantum mechanics.
\end{abstract}

Quantum chemists are throwing away important data ([11], [8]) because standard quantum mechanics does not allow their interpretation. Many-Worlds quantum mechanics $([3],[5])$ can interpret this data. Standard quantum mechanics and Many-Worlds quantum mechanics are neither mathematically nor experimentally equivalent because they differ on the meaning of the wave function $\psi$. By analyzing the unused data, quantum chemists can also make a major contribution to understanding basic reality by confirming the existence of the Many-Worlds. The last time chemistry made a central contribution to physics was in 1925, when Pauli introduced the exclusion principle in order to explain the periodic table [2], and many physicists think that chemistry is merely applied quantum mechanics. It is time chemists once again show physicists that chemistry can establish theories of physics.

I shall use Many-Worlds quantum mechanics to analyze a completely new form of reaction rate, a reaction rate whose existence has been hidden sy standard quantum mechanical analysis.

I shall start by showing that the Schrödoger equation is nothing but the mathematically consistent form of the Hamilton-Jacobi equation of classical mechanics, which is:

$$
\frac{\partial S}{\partial t}+\sum_{i=1}^{N} \frac{1}{2 m_{i}}(\vec{\nabla} S)^{2}+V(\vec{x}, t)=0
$$

where $S$ is the action. The vector $\vec{x}$ refers all the coordinates of all the particles, there being $n_{i}$ particles for each mass $m_{i}$. A particle with mass $m_{i}$ has a configuration space trajectory tangent to $\vec{\nabla}_{i} S / m_{i}$. There are an uncountable infinity of such trajectories.

In standard H-J theory, only one such trajectory is held to exist, but the equation, taken seriously [6], asserts the existence of all the trajectories. Taking the equation seriously means that a relative number density $R^{2}$ of trajectories can be defined, squared to ensure number density is non-negative, and "relative" because uncountability means if we label a countable number, we can change the number labeled by any factor $a$ without changing the physics. Labeling a countable number al- low measurements of relative density change, which is what the experiments proposed here will do. It is wellknown in fluid mechanics [12] that such relative density comparisons are possible only if the trajectories are conserved. Also, if trajectories were to disappear, it would mean that evolution past the disappearance point would not be defined, a breakdown in determinism. For both reasons, the density of trajectories must be conserved [9]:

$$
\frac{\partial \mathrm{R}^{2}}{\partial t}+\sum_{i=1}^{N} \vec{\nabla}_{i} \cdot\left(\mathrm{R}^{2} \frac{\vec{\nabla}_{i} S}{m_{i}}\right)=0
$$

Note that (2) is invariant under $R^{2} \rightarrow a R^{2}$.

If the potential is attractive, the trajectories will bend toward one another on each side of the potential center, causing a breakdown in both (1) and (2). Maxwell solved a similar equation breakdown by adding a term; we should do the same. The derivative in (1) causes the singularity, so try subtracting $\nabla_{i}^{2} R$ (linear in $R$, otherwise we get another nonlinearlty; subtract to cancel). We must however enforce density relativity by dividing by $R$ : $-\nabla_{i}^{2} R / R$. To make sure this cancels the problem in the derivative, generalize to $-\sum_{i=1}^{N}\left(1 / 2 m_{i}\right) \nabla_{i}^{2} R / R$. Finally, get the units consistent by multiplying by a constant, call it $\hbar^{2}$, with units of action-squared. The term to add to the potential in (1) is thus $-\sum_{i=1}^{N}\left(\hbar^{2} / 2 m_{i}\right) \nabla_{i}^{2} R / R$

Defining $\psi \equiv R e^{i S / \hbar}$, the two equations (1) with the new term and (2) can be written as a single equation:

$$
i \hbar \frac{\partial \psi}{\partial t}+\sum_{i=1}^{N} \frac{\hbar^{2}}{2 m_{i}} \nabla_{i}^{2} \psi-V(\vec{x}, t) \psi=0
$$

which, because it is linear, has no singularities. Equation (3) thus is the mathematically consistent form of the Hamilton-Jacobi equation. It is of course the Schrödinger equation. Notice however that the above derivation leaves us no choice in choosing the meaning of $\psi$ : it is necessarily a relative density of trajectories (worlds or universes) amplitude, not a probability amplitude. Similarly, the derivation tells us what $\hbar$ is: the strength of interaction between the worlds. It also tells us what a 
"classical" world is: a trajectory along which the other worlds can be ignored. This can occur only if $\nabla^{2} R=0$, which has $R=$ constant as the only bounded regular solution. In particular, if $V(\vec{x}, t)=0$, the classical trajectories are the plane waves, not minimal uncertainty wave packets. Many-Worlds quantum mechanics is thus more general that standard quantum mechanics, because the wave function need not be an element in a Hilbert space, which a plane wave is not. Finally, the derivation tells us why the equations of physics are quantized: to prevent singularities from arising in the laboratory.

The Many-Worlds meaning of $\psi$ entails that the Born frequencies are approached in the limit of an infinite number of experiments [9], [10].

The rate of convergence to the final Born frequency pattern is given by the Berry-Esseen Theorem [4] which applied to QM is

$$
\begin{gathered}
\left|\left[\frac{\sum_{k=1}^{l(\Delta V)} N_{k}}{N}\right]-\left[\frac{\int_{\Delta V}|\psi(\vec{x})|^{2} d \vec{x}}{\int_{V}|\psi(\vec{x})|^{2} d \vec{x}}\right]\right| \\
\leq \frac{1}{\sqrt{N}}\left(\frac{3+\sqrt{10}}{6 \sqrt{2 \pi}}\right) \times \\
{\left[\frac{\sum_{i, j}\left(\int_{V}\left|x_{i j}-\mu_{i j}\right|^{3}|\psi(\vec{x})|^{2} d \vec{x}\right)\left(\int_{V}|\psi(\vec{x})|^{2} d \vec{x}\right)^{1 / 2}}{\left(\sum_{i, j} \int_{V}\left(x_{i j}-\mu_{i j}\right)^{2}|\psi(\vec{x})|^{2} d \vec{x}\right)^{3 / 2}}\right]}
\end{gathered}
$$

First divide the total three-dimensional region $V$ into $k$ bins. When there are $N$ total electrons detected, there will be $N_{k}$ electrons in the $k$ th bin. If we add the number of electrons in all the bins up to $l(\Delta V)$, there will be $\sum_{k=1}^{l(\Delta V)} N_{k}$ in these bins, and the three-dimensional volume occupied by these bins will be $\Delta V$. If there are $m$ electrons generating the observed pattern, there will be $3 m$ coordinates for the electrons, three spatial coordinates for each of the electrons. The vector $\vec{x}$ thus denotes $\vec{x}=\left(x_{1}, x_{2}, \ldots, x_{3 m}\right)$. Thus in the last line of (4), the index $i$ labels the electrons, and $j$ labels the spatial position in rectangular coordinates $x, y, z$ of the electrons, The mean $\mu_{i j}$ of the density $\psi(\vec{x})$ is given by

$$
\mu_{i j}=\int_{V} x_{i j}\left|\psi\left(x_{1}, x_{2}, \ldots, x_{3 m}\right)\right|^{2} d^{3 m} x=\mu_{j}
$$

where the integration is once again over the entire volume where an electron might be, in general all space $R^{3}$. (In formula (4), $d^{3 m} x$ is written $d \vec{x}$.) The $j$ th spatial coordinate of the $i$ th electron is written $x_{i j}$. The last equality in (5) follows from the total antisymmetry of $\psi$ under the interchange of particles: the electrons are identical. (Thus formula (4) can be simplified, but I have expressed it as above to make clear the physical basis.) The counting of the bins must begin at bin most distance from the

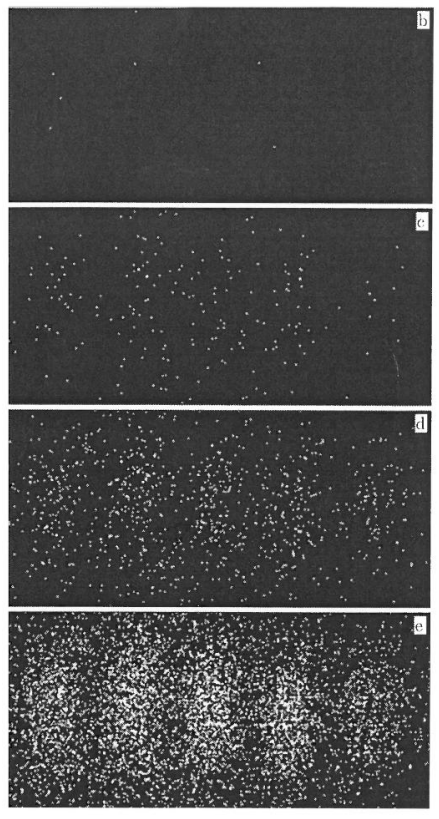

FIG. 1: Double Slit Diffraction Pattern by Electrons. The four photographs show the build-up of a double slit electron interference pattern. There are 7, 209, 1004, and 6235 electrons detected on the screen in (b)-(e) respectively. (Figure taken from [1].)

center of the distribution $\frac{1}{3 m} \sum_{i, j} \mu_{i j}=\frac{1}{3} \sum_{j} \mu_{j}$. If the data is not available in three dimensions, but only in 2-D projection (as suggested in Figure 2), then the 2-D $l(\Delta V)$ bin should be constructed of the entire $\Delta V$ along the line of sight. The Berry-Esseen constant $\left(\frac{3+\sqrt{10}}{6 \sqrt{2 \pi}}\right) \approx 0.40973$ is the known lower bound [13], but the actual constant may be larger, up to $0.5606,37 \%$ larger [7] (it is conjectured that the constant is as given in (4), but this has not been proven to date). Based on the double slit analysis [10], I do not expect this uncertainty to matter. Note that the uncertainty is less in the one-dimensional case. The double slit inequality in [10] is simpler than inequality (4), which generalizes the single particle inequality in [10] to $m$ fermions. In Figure 2, I describe how the two inequalities can be applied to test the Many-Worlds time dependent Schrödinger equation.

I hope quantum chemists will test inequality (4). Chemistry should once again be establishing fundamental physical law.
[1] Roger Bach, Damian Pope, Sy-Hwang Liou, and Herman Batelaan, "Controlled double-slit electron diffrac- tion," New Journal of Physics 15 (2013) 033018. 


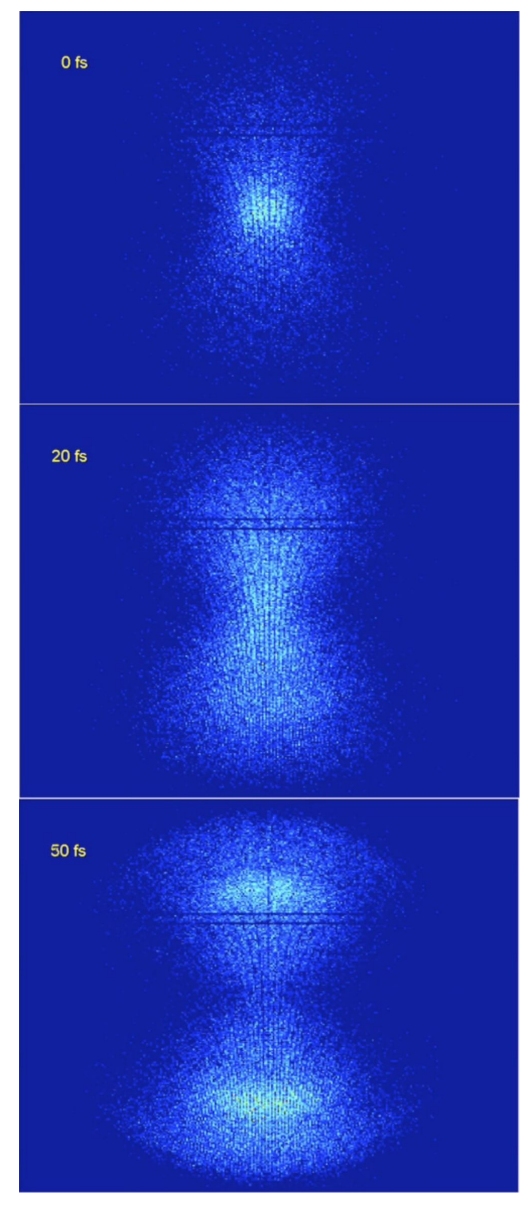

FIG. 2: Disassociation of Bromide Molecule. The three images show the final electron positions in the disassociating $\mathrm{Br}_{2}$ molecule at 0 femtoseconds, at $20 \mathrm{fs}$ and at $50 \mathrm{fs}$. The different times express the wave function of a time dependent Schrödinger equation instead of the time independent wave function represented in Figure 2. Formula 4 is time independent, so it must be applied to each of the electron distributions in the Figure. (Figure taken from the movie in [8].)
[2] Stephen G. Brush Making Twentieth Century Physics (Oxford: Oxford University Press, 2015), p. 229.

[3] Hugh Everett, "Relative State Formulation of Quantum Mechanics," Rev. Mod. Phys. 29, 454-462 (1957).

[4] William Feller, An Introduction to Probability Theory and Its Applications, Volume II (2nd ed.) (Wiley, New York, 1972), p. 542.

[5] Bryce S. DeWitt and Neill Graham The Many-Wolrds Interpretation of Quantum Mechanics (Princeton University Press, Princeton 1973), p. 185.

[6] Felix Klein "Ueber neuere englische Arbeiten zur Mechanik," Jahresbericht der Deutschen MathematikerVereinigung (Berlin: G. Reimer (1890/91)), English translation of the relevant part in The Historical Development of Quantum Theory, by Jagdish Mehra and Helmut Rechenberg, Volume 5, Part 2, p. 517 (Berlin: Springer, 1987).

[7] V. Yu. Korolev and I. G. Shevtsova, "On the Upper Bound for the Absolute Constant in the Berry-Esseen Inequality," Theory of Probability and its Applications $\mathbf{5 4}$ 638-658 (2010).

[8] Li, W., A.A. Jaroń-Beckera, C. W. Hoglea, V. Sharmaa, X. Zhoua, A. Beckera, H. C. Kapteyna, and M. M. Murnanea, "Visualizing Electron Rearrangement in Space and Time During the Transition From a Molecule to Atoms," Proc. Nat. Acad. Sci. 107, 20219-20222 (2010).

[9] Frank J. Tipler "Quantum Nonlocality Does Not Exist," Proc. Nat. Acad. Sci. doi.10.1073/PNAS.1324238111 (2014).

[10] Frank J. Tipler "Many-Worlds Quantum Mechanics is Neither Mathematically Nor Experimentally Equivalent to Standard Quantum Mechanics," ArXiv (2020).

[11] Ph. Wernet, M. Odelius, K. Godehusen, J. Gaudin, O. Schwarzkopf, and W. Eberhardt "Real-Time Evolution of the Valence Electronic Structure in a Dissociating Molecule," Phys. Rev. Lett. 103013001 (2009).

[12] Wen-Jei Yang (editor) 1989 Handbook of Flow Visualization (New York: Hemisphere Publishing) See especially Chapter 5 "Gases: Smokes" by Thomas J. Mueller, pp. 45-63.

[13] V. M. Zolotarev, "A Sharpening of the Inequality of BerryEsseen," Z. Wahrsch. Verw. Geb. 8 332-342 (1967). 